\title{
Aquaporin 3 promotes human extravillous trophoblast migration and invasion
}

\author{
Yingqi Nong ${ }^{1,2+}$, Shifen $\mathrm{Li}^{3+}$, Wenjuan Liu², Xiqian Zhang ${ }^{2}$, Lin Fan², Ye Chen², Qianwen Huang ${ }^{2}$, \\ Qianyu Zhang ${ }^{2}$ and Fenghua Liu ${ }^{2^{*}}$ (D)
}

\begin{abstract}
Problem: Does aquaporin 3 (AQP3) affect the migration and invasion of human extravillous trophoblast (HTR8/ Svneo) cells?

Method of study: A lentivirus infection system was used to construct stable cell lines with either AQP3 knockdown or overexpression. RT-PCR and western blotting were used to verify the efficiencies of AQP3 knockdown or overexpression in HTR8/Svneo cells at mRNA and protein levels, respectively. Cell Counting Kit-8 and flow cytometry assays were used to detect the influence of AQP3 knockdown or overexpression on proliferation and apoptosis of HTR8/Svneo cells. In addition, wound healing and Transwell invasion assays were used to detect the effects of AQP3 knockdown or overexpression on migration and invasion capabilities of HTR8/Svneo cells. An Agilent gene chip was used to screen for significant differentially expressed genes after AQP3 knockdown. Finally, mechanisms by which AQP3 influences the migration and invasion of HTR8/Svneo cells were explored using bioinformatic analysis.
\end{abstract}

Results: Compared with controls, migration and invasion capabilities of HTR8/Svneo cells were significantly reduced after AQP3 knockdown, and significantly increased after AQP3 overexpression. Subsequent bioinformatic analysis of gene chip expression profiles indicated downregulation of genes related to adhesion such as PDGF-B, as well as signaling pathways (such as PIK3/AKT, NF-KB, and TNF) after AQP3 knockdown.

Conclusions: AQP3 could significantly promote migration and invasion capabilities of human extravillous trophoblasts, it may mediate embryo invasion and adhesion to endometrium by regulating PDGF-B, PIK3/AKT signaling pathways, although this requires further verification.

Keywords: Aquaporin 3 (AQP3), Embryo implantation, Human extravillous trophoblast, Migration, Invasion

\section{Introduction}

Recurrent implantation failure (RIF) is one of the bottlenecks of in vitro fertilization-embryo transfer and its derivative techniques. Currently, there is no uniform definition on RIF. However, it is widely accepted as a diagnosis criteria which include an age less than 40 years and failure to achieve a clinical pregnancy after transfer

\footnotetext{
* Correspondence: liushine2006@163.com

${ }^{\dagger}$ Yingqi Nong and Shifen Li contributed equally to this work.

${ }^{2}$ Department of Reproductive Health and Infertility, Guangdong Women and Children Hospital, Guangzhou, Guangdong, China

Full list of author information is available at the end of the article
}

of at least four good-quality embryos in a minimum of three fresh or frozen cycles [1]. Incidence of RIF is up to $10-15 \%$ [2], but its pathogenesis is still unclear. Many studies have reported that two-thirds of RIF cases can be attributed to endometrial receptivity, while the other third is caused by inherent factors within embryos [3].

Embryo implantation is an important step of mammalian reproduction, and is critical for determining pregnancy. Two factors that determine embryo implantation are the embryo's implantation capability and the receiving status of the endometrium. The process of embryo

(C) The Author(s). 2021 Open Access This article is licensed under a Creative Commons Attribution 4.0 International License, which permits use, sharing, adaptation, distribution and reproduction in any medium or format, as long as you give appropriate credit to the original author(s) and the source, provide a link to the Creative Commons licence, and indicate if changes were made. The images or other third party material in this article are included in the article's Creative Commons licence, unless indicated otherwise in a credit line to the material. If material is not included in the article's Creative Commons licence and your intended use is not permitted by statutory regulation or exceeds the permitted use, you will need to obtain permission directly from the copyright holder. To view a copy of this licence, visit http://creativecommons.org/licenses/by/4.0/ The Creative Commons Public Domain Dedication waiver (http://creativecommons.org/publicdomain/zero/1.0/) applies to the data made available in this article, unless otherwise stated in a credit line to the data. 
implantation includes localization, adhesion, and invasion of the maternal endometrium until embedment into the matrix. This behavior of the embryo invading the endometrium at a specific time and space is the primitive motive for embryo implantation. The invasion process is completed when EVT differentiate from cytotrophoblasts [4]. Decidualization happens in the endometrium during the implantation window after blastocyst adhesion. Endometrial interstitial cells gradually transform into decidual stromal cells, which receive the invasion of EVT [5]. It has been shown that invasion of trophoblasts into the endometrium is similar to the metastasis of malignant tumors [6], as both involve cell invasion. Interestingly, an in vitro co-culture study reported that trophoblasts in mouse embryos exhibited stronger invasion than malignant tumor cells $[7,8]$. Directional migration of trophoblasts, which involves a series of cell signaling events, is a central step of invasion behavior. At present, widely accepted cell migration mechanisms [9] include: (1) actin depolymerization, transport of ions into cells, and increased osmotic pressure in the front of cells; (2) penetration of water through the cell membrane to increase local hydrostatic pressure, whereby the cell membrane forms local crowning including ruga, pseudopod, and vesicles; and (3) actin re-polymerization. Thus, migration speed can be controlled by osmotic pressure in extracellular medium. This means that high osmotic pressure accelerates migration, while low pressure slows down migration. During this process, AQPs play a critical role for compliance with the acceleration of intracellular and extracellular osmotic pressure changes, as well as rapid changes of cellular morphology $[10,11]$.

AQPs, a type of channel protein, can regulate levels of water and small molecular substances (such as glycerin, urea, and nitrogen), which is very important for the maintenance of body fluid equilibrium [12]. Multiple AQPs have been detected in embryos before implantation [12-14]. Expression of AQP3 lasts from the zygote to the blastula stage [15], and is the most abundant aquaporin expressed in villi during early pregnancy [16]. This suggests that AQP3 may play a role in early growth and implantation of embryos.

Our previous study found high expression of AQP3 in the cell membrane of trophoblasts in blastocysts of Kunming mice [17]. AQP3 could significantly promote both adhesion and expansion capabilities of blastocysts [18], suggesting that AQP3 participates in the process of trophoblasts invading the endometrium. However, it is unknown if AQP3 is expressed in human extravillous trophoblasts (EVT), or whether it participates in human embryo implantation. If so, the mechanism by which AQP3 regulates embryo implantation is unknown. All of these issues were addressed in the current study.

\section{Methods and materials \\ Methods \\ Cell culture}

The human trophoblast cell line HTR8/SVneo has been widely applied as an early invasion and migration model of extravillous cytotrophoblasts [19]. HTR8/SVneo, which was provided by American Type Culture Collection (USA), was cultured with Dulbecco's Modified Eagle's Medium (DMEM) containing 10\% fetal bovine serum (FBS; Gibco, USA) at $37^{\circ} \mathrm{C}$ and $5 \% \mathrm{CO}_{2}$.

\section{Construction of stable cell lines}

Plasmids containing AQP3 knockdown, AQP3 overexpression, or their respective negative control plasmids were purchased from Weijiang Biotechnology (China). Lentiviral packaging was performed in $293 \mathrm{~T}$ cells according to the manufacturer's instructions (Suzhou GenePharma, China). Lentivirus in the supernatant was collected to transfect cells. Controls were transfected with empty vector. Cells were divided into four groups: interfering AQP3 (AQP3-shRNA) group, interfering empty vector (CON-shRNA) group, overexpressing AQP3 (AQP3-OE) group and overexpressing empty vector (CON-OE) group. Cells were infected with viruses at the following multiplicities of infection (MOI): AQP3shRNA (MOI $=100)$; CON-shRNA (MOI $=100)$; AQP3$\mathrm{OE}(\mathrm{MOI}=150)$; CON-OE $(\mathrm{MOI}=100)$. Strict phenotype selection was performed on stably infected HTR8/ SVneo cells with $0-10 \mu \mathrm{g} / \mathrm{mL}$ puromycin (MPbio, USA) to use resistance as a screening index. Furthermore, cells were stably cloned in $0.5 \mu \mathrm{g} / \mathrm{mL}$ puromycin.

\section{RNA isolation, CDNA synthesis and qRT-PCR analysis}

Total RNA was extracted from HTR8/Svneo cells according to the instructions of a TRIzol kit (Taraka Biotechnology, China), and measured with a spectrophotometer (Nanodrop 2000; Thermo Scientific, USA).

cDNA synthesis The annealing mixture contained $1 \mu \mathrm{g}$ of RNA, $1 \mu \mathrm{l}$ of $0.5 \mu \mathrm{g} / \mathrm{ul}$ Oligo (dT) $18,1 \mu \mathrm{l}$ of dNTPs Mix $(2.5 \mathrm{mM})$, and RNase-free water to a total volume of $10 \mu \mathrm{l}$. Then, it was incubated in a thermal cycler at $65^{\circ} \mathrm{C}$ for $5 \mathrm{~min}$ and placed on ice for $1 \mathrm{~min}$ at least. The contents of the tube were collected by brief centrifugation before the following were added to the tube: Random primer, $1 \mu \mathrm{l}, 5 \times$ Buffer, $4 \mu \mathrm{l}$, RNase Inhibitor, $0.5 \mu \mathrm{l}$, and RNase Free $\mathrm{H} 2 \mathrm{O} 4.5 \mu \mathrm{l}$. The pipette was gently sucked and tapped several times to obtain a better mixture. Incubation was done as follows: $10 \mathrm{~min}$ at $30^{\circ} \mathrm{C}, 60 \mathrm{~min}$ at $42^{\circ} \mathrm{C}$ and then $15 \mathrm{~min}$ at $70^{\circ} \mathrm{C}$. The cDNA synthesis reaction was stored at $-20^{\circ} \mathrm{C}$, or PCR was performed immediately. 
RT-qPCR reactions were conducted with a QuantStudio 5 real-time PCR system (Thermo Fisher Scientific, Waltham, MA USA). The primers were designed by Primer-BLAST (NCBI). The primer sequences and PCR product sizes were detailed in Table 1. The real time PCR mixture contained SYBR Green Premix, $10 \mu$ l, Forward Primer $(10 \mu \mathrm{M}), 0.4 \mu \mathrm{l}$, Reverse Primer $(10 \mu \mathrm{M})$, $0.4 \mu \mathrm{l}$, Template, $1.2 \mu \mathrm{l}$, ROX Reference Dye II (50×), $0.4 \mu \mathrm{l}$, DNA template $2.0 \mu \mathrm{l}$, and RNase Free $\mathrm{H} 2 \mathrm{O}$ to a total volume of $20 \mu \mathrm{l}$. After the solution was mixed and centrifuged at 5000 RPM for a short time, the reaction mixtures $(8 \mu \mathrm{l})$ were added into a 384-well PCR plate, and the cDNA samples $(2 \mu \mathrm{l})$ were added. The plate was sealed and placed on ice. The PCR was initiated by heating the mixture to $95^{\circ} \mathrm{C}$ for $5 \mathrm{~min}$, followed by 40 cycles of $15 \mathrm{~s}$ at $95^{\circ} \mathrm{C}$ and $60 \mathrm{~s}$ at $60^{\circ} \mathrm{C}$ and then $15 \mathrm{~s}$ at $95^{\circ} \mathrm{C}$. To establish the melting curve, the mixture was heated to $95^{\circ} \mathrm{C}$ for $10 \mathrm{~s}, 60^{\circ} \mathrm{C}$ for $60 \mathrm{~s}$, and $95^{\circ} \mathrm{C}$ for $15 \mathrm{~s}$ sequentially after the amplification reaction was over. The $2^{-\triangle \Delta C T}$ method was used to quantify relative expression of AQP3 mRNA. Each real-time PCR included a notemplate control. The experiments were repeated 3 times with triplicates of each sample.

\section{Western blotting}

Cells were collected and lysed with RIPA lysis buffer and phenylmethylsulfonyl fluoride (Beyotime Biotechnology, China) on ice for $30 \mathrm{~min}$, quantified by bicinchoninic acid assay. Equal amounts of protein $(\sim 10 \mu \mathrm{g})$ were separated by $10 \%$ sodium dodecyl sulfate polyacrylamide gel electrophoresis (SDS-PAGE) and loaded for SDS-PAGE electrophoresis. After transfer to a polyvinylidene fluoride membrane, the membrane was blocked in 5\% skim milk, sealed for $1 \mathrm{~h}$ at room temperature with shaking, and incubated with a primary antibody over-night at $4{ }^{\circ} \mathrm{C}$. The primary antibodies used were rabbit polyclonal anti-AQP3 antibody (1:1000, Abcam, UK, ab125219), rabbit polyclonal anti-PDGFB antibody (1:1000, Cohesion, UK, CPA1865). IgG from rabbit serum (1:1000, Sigma, USA, I5006) was used for negative control.

Table 1 The Primer sequences and the product size of target and control genes

\begin{tabular}{lll}
\hline Primer & Primer sequence (5'-3') & size (bp) \\
\hline AQP3 & $\begin{array}{l}\text { Forward primer: ACCATCAACCTGGCCTTGG } \\
\text { Reverse primer: GGGGACGGGGTGTTGTA }\end{array}$ & 390 \\
PDGFB & $\begin{array}{l}\text { Forward primer: ACTGATGGGGTCGCTCTTG } \\
\text { Reverse primer: CAGGGATCAGGCAGGCTATG }\end{array}$ & 126 \\
FOS & $\begin{array}{l}\text { Forward primer: GTGCCAACTTCATTCCCACG } \\
\text { Reverse primer: GGCCTCCTGTCATGGTCTTC }\end{array}$ & 186 \\
SNAIL1 & $\begin{array}{l}\text { Forward primer: CCTGTCTGCGTGGGTITTG } \\
\text { Reverse primer: ACCTGGGGTGGATATTGC }\end{array}$ & 198 \\
GAPDH & $\begin{array}{l}\text { Forward primer: GAAGCTCATTCCTGGTATGACA } \\
\text { Reverse primer: GGGAGATTCAGTGTGGTGG }\end{array}$ & 189 \\
\hline
\end{tabular}

Finally, the membrane was incubated with horseradish peroxidase-labeled secondary antibody $(120,000$, Boster Biological Technology, China, BA1054) at room temperature for $1 \mathrm{~h}$, and developed by enhanced chemiluminescence. The experiments were repeated 3 times. Image Pro-Plus 6.0 software (Media Cybernetics, USA) was used to analyze gray values.

\section{Cell proliferation/CCK-8 assay}

HTR8/Svneo cells $\left(100 \mu \mathrm{L} ; 1 \times 10^{5}\right.$ cells $\left./ \mathrm{mL}\right)$ in vector control, knockdown, and overexpression groups were added into 96-well plates in triplicate, and cultured at $37^{\circ} \mathrm{C}$ overnight. CCK- 8 kit reagent $(10 \mu \mathrm{L}$; Dojindo, Japan) was added into each well and incubated for $2 \mathrm{~h}, 3$ $\mathrm{h}$, and $4 \mathrm{~h}$. Optical density at $450 \mathrm{~nm}$ of each well was detected each time point with a multifunctional microplate reader.

\section{Flow cytometry assay}

Annexin V-APC/7-AAD double staining was performed on HTR8/Svneo cells according to the instructions of an Annexin V-APC/7AAD Apoptosis Detection Kit [Multisciences (Lianke) Biotech, China]. Live, early apoptotic, and late apoptotic or necrotic cells were classified using flow cytometry (AccuriC6, Becton Dickinson, USA) and Flow Jo 7.6.1 software (FlowJo, USA).

\section{Wound healing assay}

HTR8/Svneo cells $\left(1 \times 10^{6}\right.$ cells $\left./ \mathrm{mL}\right)$ were seeded in a six-well plate and routinely cultured in an incubator. When cells grew into a monolayer, they were treated with mitomycin for $1 \mathrm{~h}$ to inhibit cell division. Next, a sterile $10 \mu \mathrm{l}$-pipette tip was used to scrape cell culture plates. Scraped cells were washed twice with phosphatebuffered saline, cultured in serum-free medium, incubated in an incubator, and photographed at $0,6,24$ and $48 \mathrm{~h}$ after scratching. Image Pro-Plus 6.0 was used to measure scratch depth at any five sites at the same time point to calculate migration rates, thus reflecting cell mobility and migratory capabilities.

\section{Transwell invasion assay}

A Transwell invasion system $(8-\mu \mathrm{m}, 24-$ well; Corning, UK) coated with Matrigel ( $40 \mu \mathrm{L}$; Becton Dickinson) was used. Briefly, $1 \times 10^{5}$ cells were suspended in DMEM without serum, and seeded in the upper chamber. DMEM containing 10\% FBS was then added to the lower chamber and the plate was incubated at $37^{\circ} \mathrm{C}$ and $5 \%$ $\mathrm{CO}_{2}$. After $24 \mathrm{~h}$, cells were fixed with methanol and stained with $0.1 \%$ crystal violet. The quantity of colored cells in five random visual fields was counted using an inverted microscope (Nikon, Japan). 


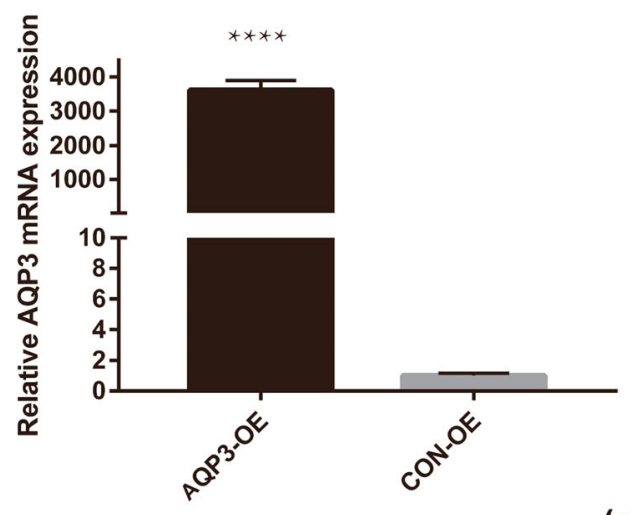

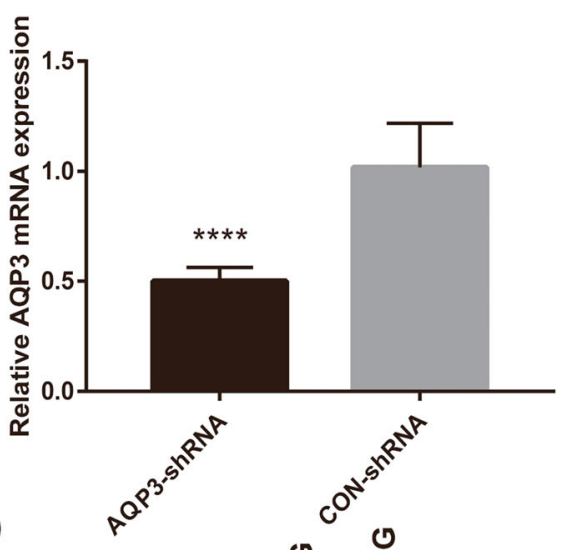

(a)

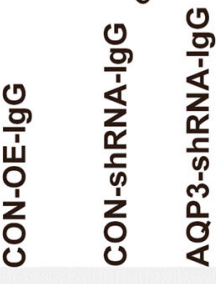

AQP3

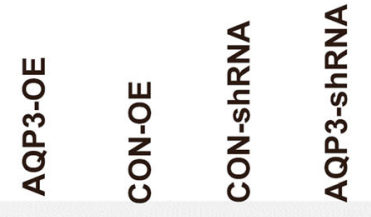

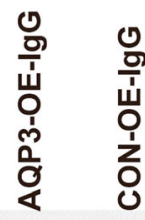

GAPDH
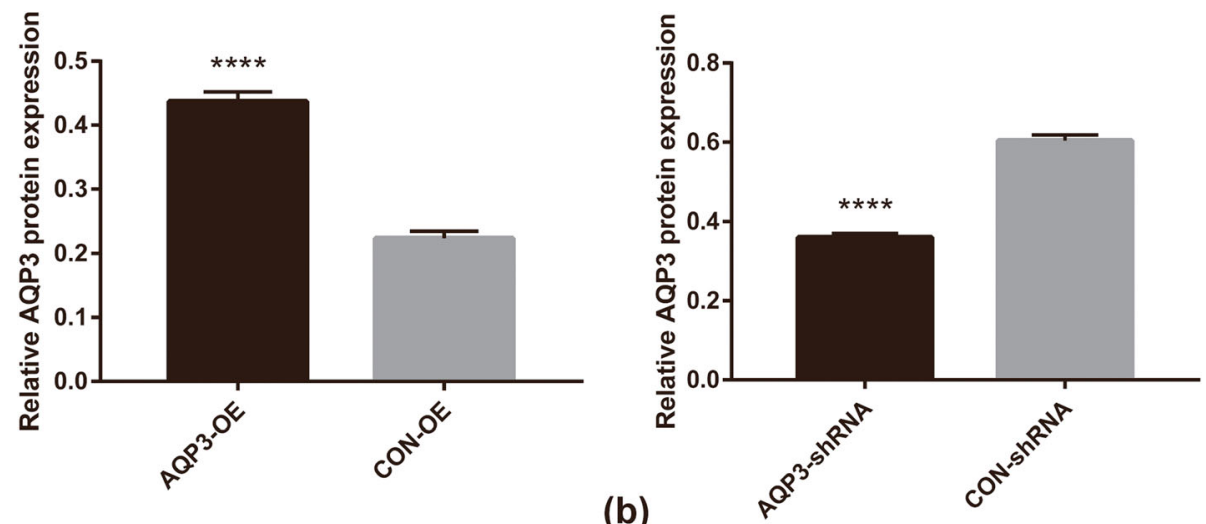

(b)

Fig. 1 Verification of AQP3 knockdown and overexpression efficiency. (a) Relative expression levels of AQP3 mRNA in AQP3 knockdowned and overexpressed HTR8/SVneo. (b) Relative expression levels of AQP3 protein in AQP3 knockdowned and overexpressed HTR8/SVneo. Suggesting effective AQP3 knockdown or overexpression in HTR8/SVneo. ${ }^{* *} P<0.01 ;{ }^{* * *} P<0.0001$

Table 2 Apoptosis rates of HTR8/SVneo cells. The apoptotic cells include late apoptotic (Q2:APC+/7AAD) and early apoptotic cells (Q3:APC+/7AAD-)

\begin{tabular}{llc}
\hline Group & Apoptosis rates (Mean \pm SEM) & $\boldsymbol{p}$-value \\
\hline AQP3-shRNA & $7.157 \% \pm 4.391 \%$ & $<0.05$ \\
CON-shRNA & $5.36 \% \pm 2.594 \%$ & \\
AQP3-OE & $4.727 \% \pm 1.984 \%$ & $>0.05$ \\
CON-OE & $5.28 \% \pm 0.1353 \%$ & \\
\hline
\end{tabular}

\section{Whole genome expression profile}

The Gene expression profiles assay was performed according to a previously described method [20]. Gene expression profiles of AQP3-shRNA and CON-shRNA were analyzed by two-color gene expression microarray (Agilent Technologies, USA) according to the instructions of a Low Input Quick Amp Labeling Kit TwoColor (Agilent). Total RNA obtained in the extraction phase was used as a template, and the first strand of cDNA was reverse transcribed using T7 RNA polymerase. The second strand of cDNA was used as the synthesis template to perform in vitro transcription and promote generation of cRNA. An Agilent cRNA labeling 

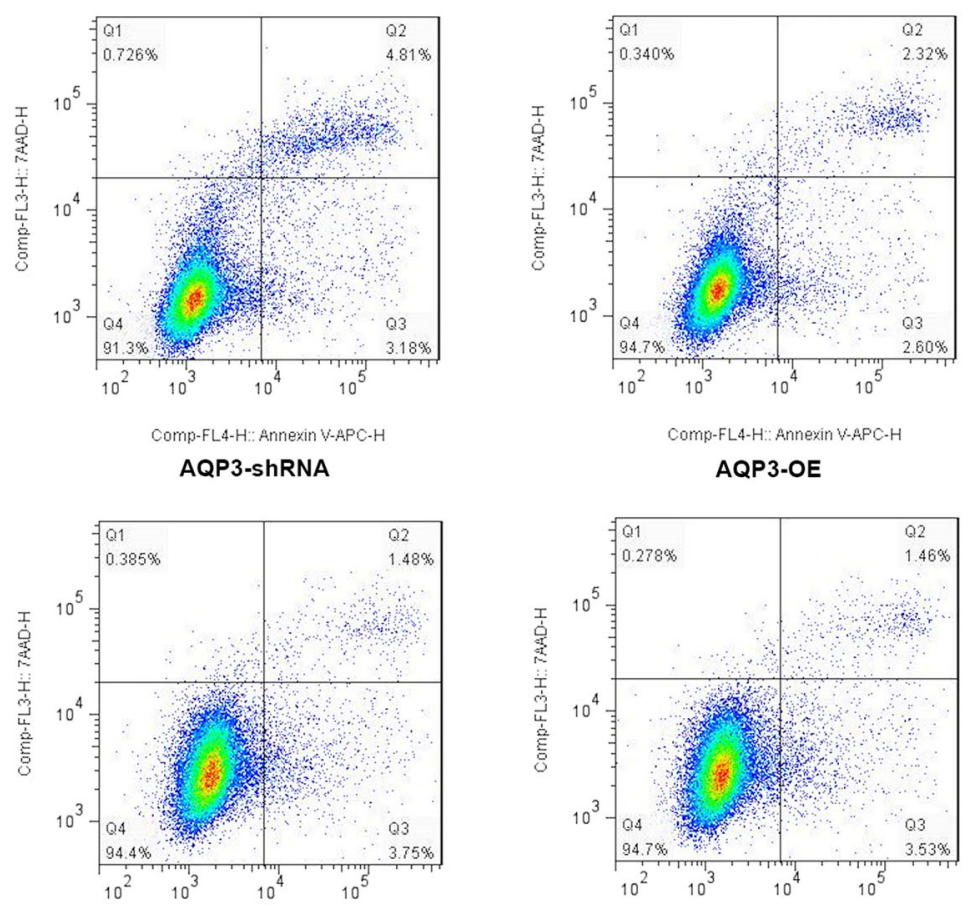

Comp-FL4-H:: Annexin V-APC-H

CON-shRNA

(a)

CON-OE
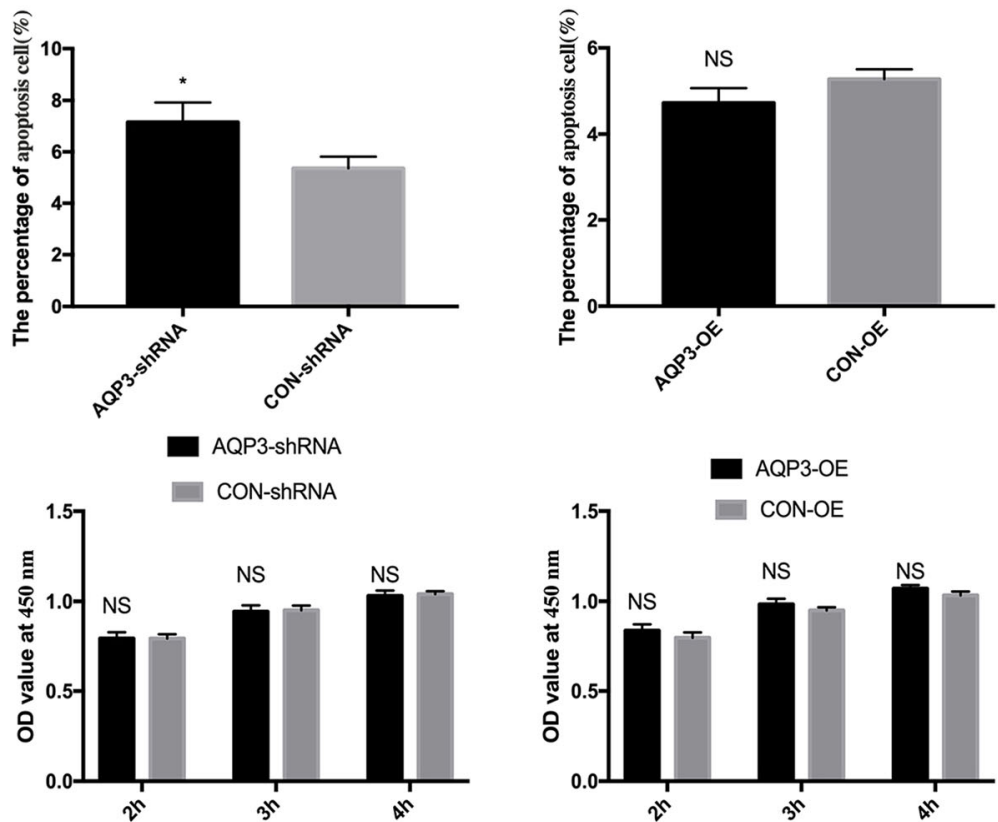

(b)

Fig. 2 Flow cytometry and CCK-8 assays. (a) Apoptosis rates of HTR8/SVneo cells as analyzed by flow cytometry. (b) Proliferation rates as detected by CCK-8 assay. AQP3 knockdown expression increased the apoptosis of HTR 8/SVneo, but had not effect on cell proliferation. Overexpression of AQP3 had no effect either on the cell apoptosis or proliferation rates. ${ }^{*} P<0.05, \mathrm{NS}: P>0.05$ 
Table 3 Proliferation rates of HTR8/SVneo cells

\begin{tabular}{llll}
\hline Time (after CCK-8 addition) & \multicolumn{2}{l}{ Proliferation rates (Mean \pm SEM) } & \multicolumn{1}{c}{ p-value } \\
\cline { 2 - 3 } & AQP3-shRNA & CON-shRNA & $>0.05$ \\
\hline $2 \mathrm{~h}$ & $79.3 \% \pm 3.5 \%$, & $79.3 \% \pm 2.3 \%$ & $>0.05$ \\
$3 \mathrm{~h}$ & $94.3 \% \pm 3.5 \%$ & $95.0 \% \pm 2.6 \%$ & $>0.05$ \\
$4 \mathrm{~h}$ & $100.3 \pm 3.0 \%$ & $100.4 \% \pm 1.7 \%$ & $\mathbf{p}$-value \\
Time (after CCK-8 addition) & Proliferation rates (Mean \pm SEM) & & $>0.05$ \\
& AQP3-OE & CON-OE & $>0.05$ \\
$3 \mathrm{~h}$ & $83.7 \% \pm 3.5 \%$ & $79.7 \% \pm 3.1 \%$ & $>0.05$ \\
$4 \mathrm{~h}$ & $98.3 \% \pm 3.1 \%$ & $95.0 \% \pm 1.7 \%$ & \\
\hline
\end{tabular}

kit was used to incorporate cRNA with $\mathrm{Cy}-3$, which allowed purification and qualification of cRNA (Nanodrop 2000). After hybridization, washing, and chip scanning, data were extracted to perform bioinformatic analysis using Agilent Feature Extraction Software. Doing q-PCR verfication for FDGF-B, FOS and Snail1, which showed significantly decrease in the results of the gene expression profile experiment.

\section{Statistical analysis}

Data were analyzed by SPSS 23.0 software (IBM, USA). Each experiment was performed in triplicate, and data were expressed as mean \pm standard deviation (SD). Migration rate and invasion index were analyzed using a two independent-samples t-test. Proliferation and apoptosis rates were analyzed by analysis of variance. $P<0.05$ was considered statistically significant.

\section{Results}

\section{Verification of AQP3 knockdown and overexpression} efficiency

After construction of stable cell lines, AQP3 knockdown and overexpression efficiencies at mRNA and protein levels in HTR8/Svneo cells were detected using RT-PCR and western blotting, respectively. The results indicated that compared with the CON-shRNA group, AQP3

Table 4 Migration rates of HTR8/Svneo cells

\begin{tabular}{llll}
\hline \multirow{2}{*}{ Time (after scratch) } & \multicolumn{2}{l}{ Migration rates (Mean \pm SEM) } & p-value \\
\cline { 2 - 3 } & AQP3-shRNA & CON-shRNA & \\
\hline $6 \mathrm{~h}$ & $20.96 \% \pm 0.4032 \%$ & $35.22 \% \pm 1.099 \%$ & $<0.0001$ \\
$24 \mathrm{~h}$ & $43.29 \% \pm 0.5446 \%$ & $57.79 \% \pm 1.531 \%$ & $<0.0001$ \\
$48 \mathrm{~h}$ & $57.32 \% \pm 0.5719 \%$ & $86.57 \% \pm 0.8777 \%$ & $<0.0001$ \\
Time (after scratch) & Migration rates (Mean \pm SEM) & p-value \\
& AQP3-OE & CON-OE & \\
$6 \mathrm{~h}$ & $40.35 \% \pm 2.074 \%$ & $34.95 \% \pm 0.7426 \%$, & $<0.05$ \\
$24 \mathrm{~h}$ & $80.31 \% \pm 1.425 \%$ & $60.59 \% \pm 1.425 \%$ & $<0.0001$ \\
$48 \mathrm{~h}$ & $99.61 \% \pm 0.2702 \%$ & $87.34 \% \pm 1.09 \%$ & $<0.0001$ \\
\hline
\end{tabular}

knockdown resulted in downregulation of AQP3 mRNA expression by $50.8 \%(P<0.0001)$, and downregulation of AQP3 protein levels by $40.3 \%(P<0.0001)$. Compared with the CON-OE group, AQP3 overexpression resulted in upregulation of AQP3 mRNA expression by 3579-fold $(P<0.0001)$, and upregulation of AQP3 protein levels by 2-fold $(P<0.0001)$ (Fig. 1).

AQP3 knockdown expression increased the apoptosis of HTR 8/SVneo cells but had no significant effect on their cell proliferation

Apoptosis rates of HTR8/SVneo cells were analyzed by flow cytometry while proliferation rates were detected by CCK-8 assay. Q2 (APC+/7AAD+) indicates late apoptotic/necrotic cells, Q3 (APC+/7AAD-) indicates early apoptotic cells, Q4 (APC-/7AAD-) indicates living cells. The apoptotic cells include late apoptotic and early apoptotic cells $(\mathrm{Q} 2+\mathrm{Q} 3)$. Compared with the control, apoptosis in the AQP3-shRNA group was significantly upregulated by $33.5 \%(P<0.05)$. However, the rate of apoptosis in HTR8/Svneo cells in the AQP3-OE group was downregulated by $11.8 \% \quad(P>0.05)$ (one-way ANOVA) (Table 2, Fig. 2a). Proliferation rates in AQP3shRNA, CON-shRNA, AQP3-OE, and CON-OE groups at $2 \mathrm{~h}, 3 \mathrm{~h}, 4 \mathrm{~h}$ after CCK-8 addition were not significantly different $(P>0.05)$. (two-way ANOVA). (Table 3, Fig. 2b).

\section{Migratory rate and invasive ability of HTR8/Svneo cells were increased after overexpressing AQP3 while be reduced after knockdown of AQP3}

Wound healing assay show that at $6 \mathrm{~h}$ after scratch, cell migration rate in the AQP3-shRNA group was downregulated by $40.50 \%$ compared with the CON-shRNA group $(P<0.0001)$, while AQP3-OE group was upregulated by $15.44 \%$ compared with the CON-OE group $(P<0.05)$. At $24 \mathrm{~h}$ after scratch, migration rate of AQP3-shRNA was downregulated by $25.09 \%$ compared with CON-shRNA $(P<0.0001)$, while AQP3-OE group was upregulated by $32.56 \%$ compared with the CON-OE group $(P<0.0001)$. At $48 \mathrm{~h}$ after scratch, migration rate of AQP3-shRNA 


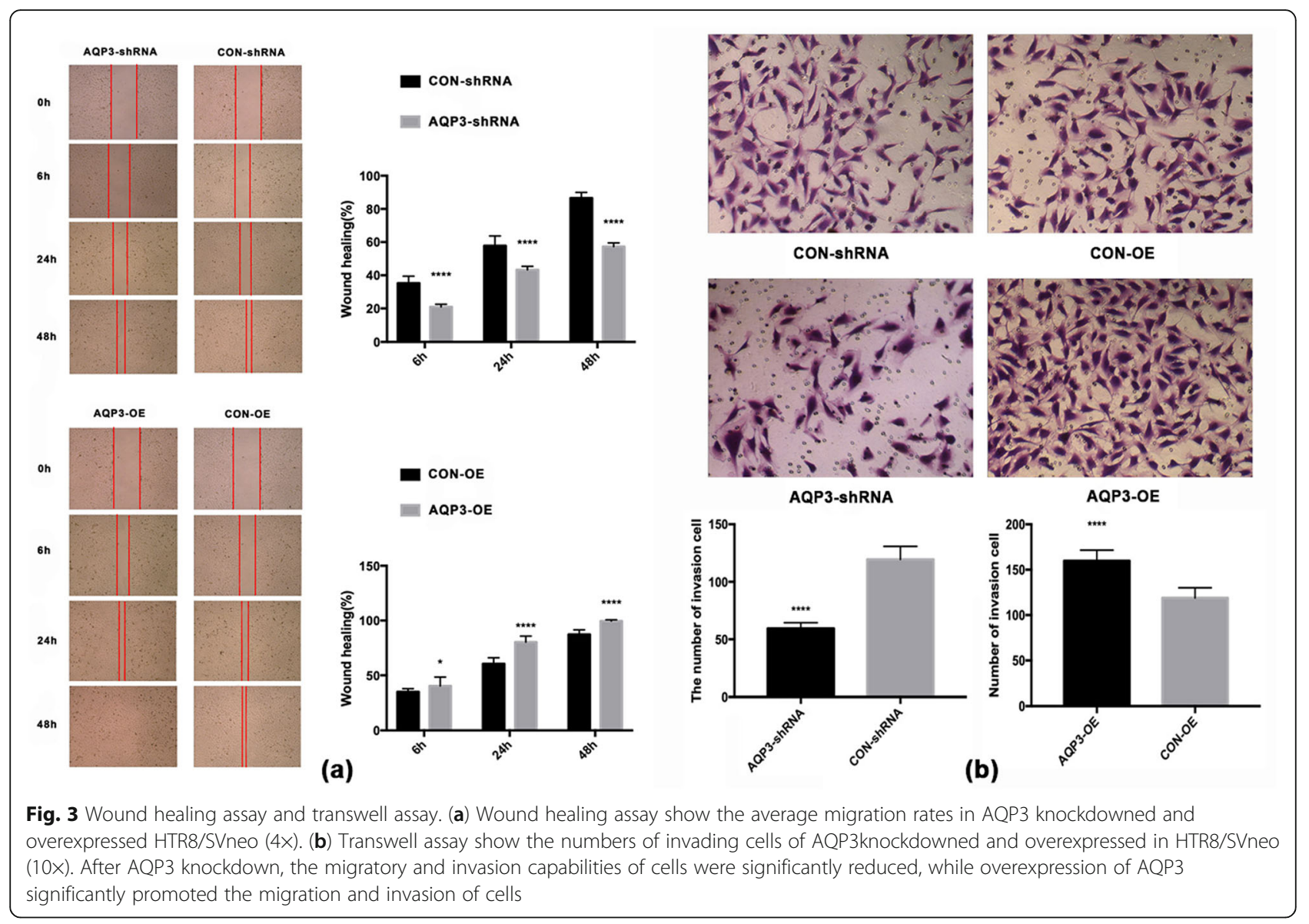

was downregulated by $33.79 \%$ compared with $\mathrm{CON}$ shRNA $(P<0.0001)$, while AQP3-OE group was upregulated by $14.05 \%$ compared with the CON-OE group $(P<$ 0.0001) (Table 4, Fig. 3a). After AQP3 knockdown, numbers in the AQP3-shRNA group were downregulated by $50.25 \%$ compared with the CON-shRNA group $(P<$ 0.0001). After AQP3 overexpression, numbers in the AQP3-OE group were upregulated by $34.38 \%$ compared with the CON-OE group $(P<0.0001)$ (t-test), $(\times 10$ amplification) (Table 5, Fig. 3b).

\section{Whole genome expression profile}

To further study signaling pathways regulated by AQP3 during embryo implantation, an Agilent gene expression microarray was used to examine AQP3-shRNA and

Table 5 Numbers of invading cells in HTR8/Svneo cells

\begin{tabular}{lll}
\hline group & invading cells (Mean \pm SEM) & p-value \\
\hline AQP3-shRNA & $59.4 \pm 1.29$ & $<0.0001$ \\
CON-shRNA & $119.4 \pm 2.952$ & \\
AQP3-OE & $159.7 \pm 3.046$ & $<0.0001$ \\
CON-OE & $118.9 \pm 2.914$ & \\
\hline
\end{tabular}

CON-shRNA groups. Genes with differential expression fold-changes $\geq 2$ and $\mathrm{q}<0.05$ as screened with Significance Analysis of Microarrays software were taken as significant differentially expressed genes. The results indicated that after AQP3 gene downregulation, there were 311 significant differentially expressed genes (150 upregulated and 161 downregulated) (Fig. 4). The result of gene ontology $(\mathrm{GO})$ analysis of genes with differential expression (fold-change $>2$ ) between AQP3 knockdown and its control group indicated these genes involved in angiogenesis, cell migration, inflammatory response, cell adhesion, and extracellular matrix recombination. Among them, 11 differentially expressed genes were related to cell migration (GO: 0030335) were notably downregulated $(P=0.000119)$, resulting in some critical factors (e.g. PDFGF-B, FOS and SNAIL1). Fourteen differentially expressed genes related to cell adhesion (GO: 0007155), such as ICAM-1, COL18A, and JUP, were significantly downregulated $(P=0.00345)$ (Fig. 5a, b; Fig. 6). Screening of differentially expressed genes by Kyoto Encyclopedia of Genes and Genomes (KEGG) enrichment analysis revealed participation primarily in tumor cell metastasis, adhesion, and apoptosis, as well as MAPK, PIK3-AKT, cell adhesion-related, tumor necrosis 


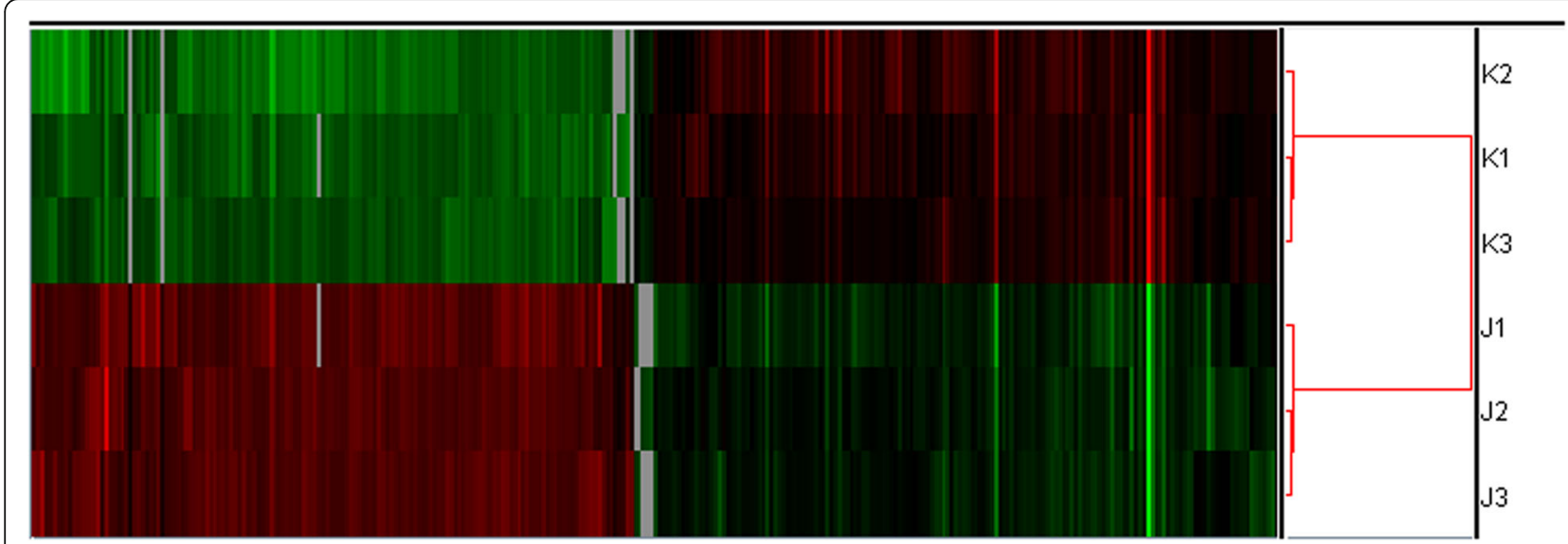

Fig. 4 Clustering gene expression patterns. J: AQP3-shRNA, K: CON-shRNA. Red represents gene upregulation, while green represents gene downregulation

factor (TNF), and NF- $\mathrm{kB}$ signaling pathways. Among them, the majority of genes involved in cell migration, adhesion, PIK3 and NF- $\mathrm{B}$ B signaling pathway were down-regulated, and AQP3-shRNA was significantly down-regulated compared with CON-shRNA, with statistically significant differences (Fig. 5c, Supplementary Table). Of all these pathways, changes in cell migration and adhesion-related signaling were the most significant.

\section{To verify the expression of some differentially expressed} genes selected from the results of whole genome expression profile

The results of q-PCR verfication for FDGF-B,FOS and Snaill showed that, the mRNA expression level of FDGF-B in AQP3-shRNA group was significantly lower than that in CON-shRNA group $(P<0.0001)$, while FOS,
Snail1 mRNA expression were lower than that of CONshRNA group, but there were no significant difference ( $P$ values were 0.068 and 0.168 , respectively) (Fig. $7 \mathrm{a}$ ). The protein level of FDGF-B was further verified, Western blot showed that the FDGF-B protein level in AQP3-shRNA group was significantly lower than that in CON-shRNA group $(\mathrm{P}<0.0001)$ (Fig. $7 \mathrm{~b})$.

\section{Discussion}

It is becoming increasingly evident that AQP3 is expressed in multiple malignant tumor cells [21] and participates in both tissue oncogenicity and tumor cell migration [22-26]. The invasion process of the trophoblast into the maternal endometrium and metastasis of malignant tumors both rely on cell invasion behaviors. However, mechanisms by which AQP3 influences

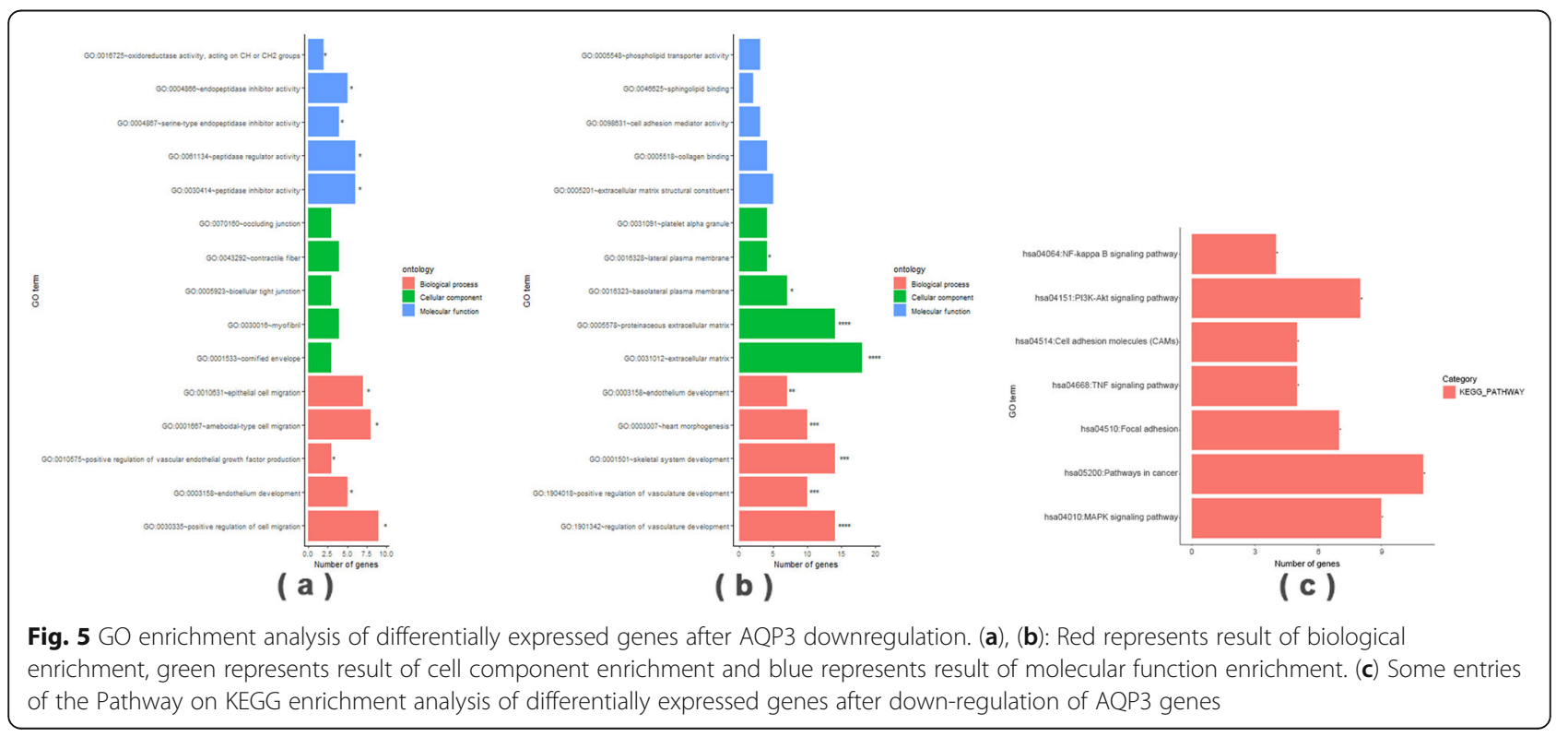




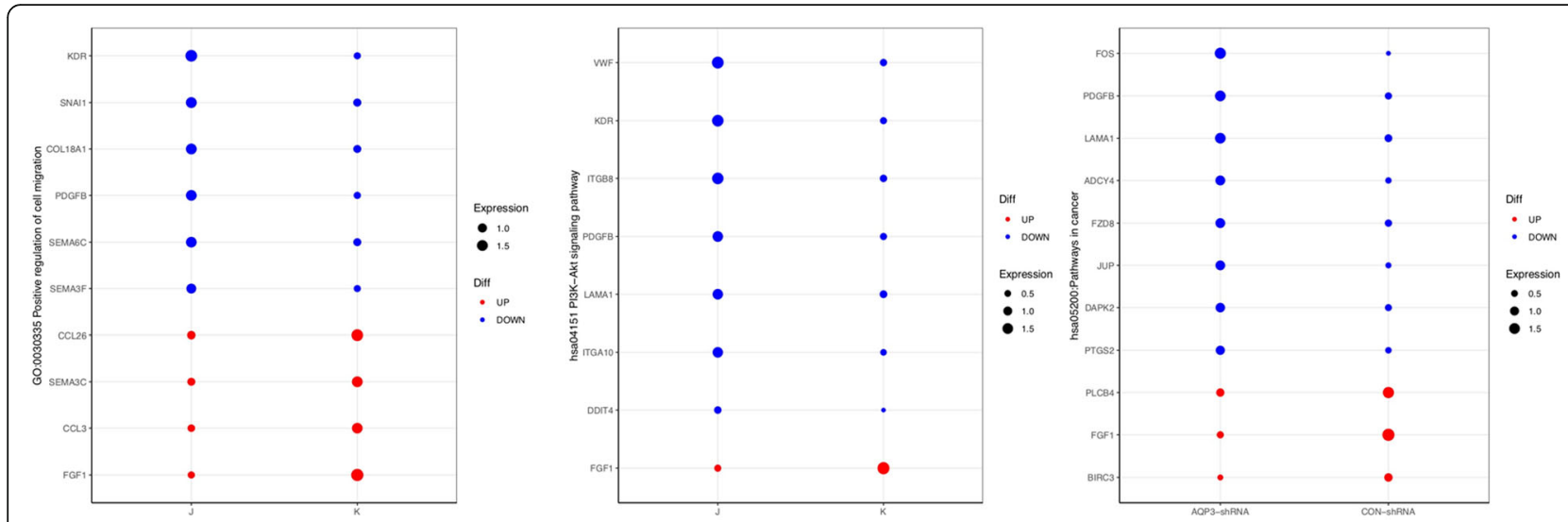

Fig. 6 KEGG enrichment analysis of cellular pathways associated with differentially expressed genes after AQP3 downregulation. Expression abundance of genes involved in cell migration, adhesion, PIK3, and NF-KB signaling pathway J: AQP3-shRNA, K: CON-shRNA. The size of each dot represents expression abundance, while difference is shown by color: blue, down-regulated significantly; red, up-regulated significantly

human reproduction are not as clear as those by which malignant tumor metastasis is promoted. Currently, most investigations on embryo implantation focus on this interaction from the aspect of endometrial receptivity. A recent study [27] suggested that AQP3 might enhance endometrial receptivity to facilitate embryo implantation, whereas few investigate the embryo's capacity for implantation. There is no study at present to clarify the molecular mechanism by which AQP3 acts on embryo to facilitate its' implantation.

In a previous study reported expression of AQP3 was reported in four-cell, eight-cell, morula, and blastula stages of mouse embryos, with the highest expression on the membrane of blastula-stage trophoblasts [17]. Further in vitro study [18] showed that, HB-EGF could enhance AQP3 expression in blastocysts in a dosedependent manner, thereby promoting the attachment and outgrowth of blastocyst trophoblast cells, and migth mediate its extension and migration through the regulation of $\mathrm{HB}-\mathrm{EGF} / \mathrm{EGFR} / \mathrm{ERK} / \mathrm{AQP} 3$ signaling pathway. However, the downstream pathway of AQP3 is not clarified.

In the present study, the human HTR8/Svneo cell line was used as model of early embryonic EVT invasion and migration to construct AQP3 knockdown and overexpression cells. The results indicate that overexpression of AQP3 significantly promoted the migration and invasion of cells, in aggreement with the results of Huang et al. [24] After AQP3 knockdown, the migratory and invasion capabilities of cells were significantly reduced, and apoptosis was significantly upregulated, supporting the results of previous reports $[17,18]$, Reca Alejandra et al. [28] and Xiong et al. [29] .

To explore more comprehensively the signaling mechanisms of AQP3 in promoting EVT migration, this study screened shRNA interfering AQP3 and undisturbed HTR8/SVneo cells by bioinformatics analysis gene chip expression spectrum experiment. Of the 311

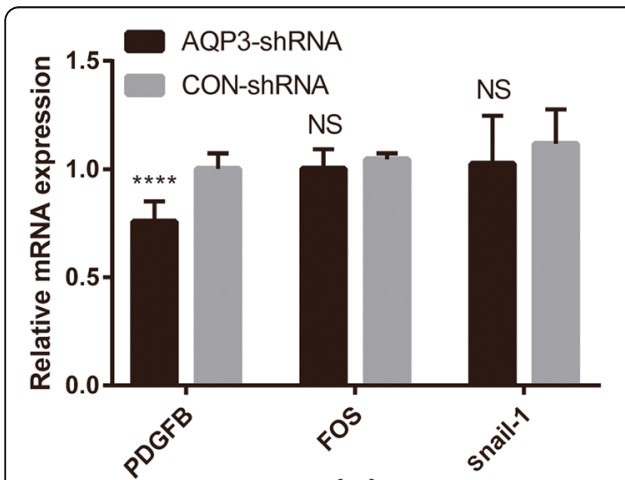

(a)
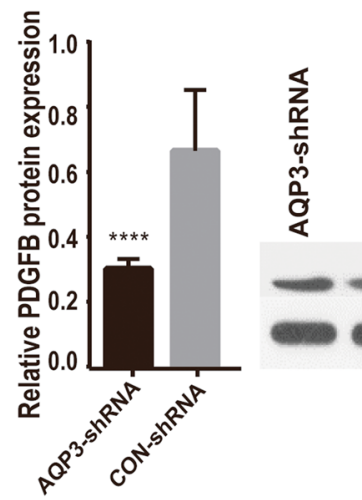

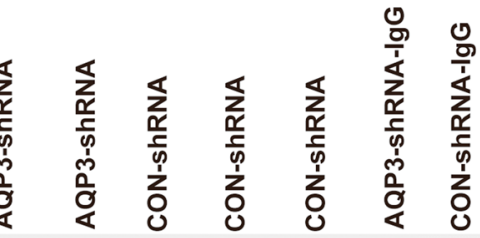

PDGFB

GAPDH

Fig. 7 PDGF-B, FOX, Snail1 expression in HTR8/Svneo cells. (a) The mRNA relative expression of PDGF-B, FOX, Snail1 in the AQP3 knockdown cells. (b) The PDGF-B protein level in the AQP3 knockdown cells 
differentially expressed genes screened, 150 were upregulated and 161 were downregulated. GO analysis and GO enrichment analysis found that these genes are mainly involved in angiogenesis, cell migration, inflammatory response, cell adhesion, extracellular matrix recombination. Bioinformatics analysis of gene chip expression profiling data reveals that key genes related to migration, such as platelet-derived growth factor-B (PDGF-B), Snai1, and FOS, were notably down-regulated after AQP3 knockdown expression.

Further q-PCR validation of these two key genes revealed that PDGF-B mRNA expression was prominently downregulated after knockdown expression, while Snai1, FOS mRNA expression was non-significantly downregulated. PDGFB protein levels were also observably downregulated.

PDGF is a dimeric molecule existing as homodimers or heterodimers of related polypeptide chains (A and B). PDGF-B is a member of the PDGF family, which has the ability to link with cystine and play a crucial role in development, cell proliferation, cell survival, and angiogenesi s[30]. PI3K/AKT, JNK, and PLC $\gamma$ pathways were involved in the process of PDGF-B binding to PDGFR $\beta$ and inducing receptor dimerizatio $\mathrm{n}[31]$. Schwenke et.a 1[32]. found that PDGF-B trigger undirected motility in endometrial stromal cells, while pathway inhibitor-based studies have shown that ERK1/2, PI3K/Akt and p38 signaling are associated with chemotactic motility, whereas chemokines (PDGF-B) are mainly dependent on PI3 kinase/Akt activation. Jing et al .333 found that PDGFB and PI3K/AKT signaling pathways have co-expression networks together with the false detection rate is very low, and PDGFB promote the metastasis of oral squamous cell carcinoma through the PI3K/AKT signaling pathway. In this study, the differentially expressed genes screened KEGG pathway analysis are mainly involved in tumor-related pathways, MAPK pathways, PI3K-AKT pathways, and NF-B signaling pathways. PI3K-AKT pathways have also been identified as closely related to tumor cell migration. Therefore, we speculate that AQP3 may play a role by acting on PDGF-B gene and $\mathrm{PI} 3 \mathrm{~K} / \mathrm{AKT}$ signaling pathways that mediate the migration and invasion of extravillous trophoblastic cells, thereby mediating embryo implantation. However, further investigation is needed to confirm this conjecture.

\section{Conclusions}

Collectively, these results reveal that AQP3 is an important positive regulatory factor for fetal-maternal crosstalk during the first trimester of pregnancy, whereby it may act on PDGF-B gene to promote migration and invasion ability of trophoblast cells, the underlying mechanism still requires a further investigation.

\section{Abbreviations}

AQP3: Aquaporin3; RIF: Recurrent implantation failure; EVT: Extravillous trophoblasts; EMT: Epithelial-mesenchymal transition; HB-EGF: Heparinbinding epidermal growth factor-like growth factor

\section{Supplementary Information}

The online version contains supplementary material available at https://doi. org/10.1186/s12958-021-00726-z.

Additional file 1. Supplementary table. Functional categories of selected genes differentially expressed in the HTR8/Svneo cells AQP3shRNA and CON-shRNA.

\section{Acknowledgements}

We are particularly grateful to the Medical Transformation Center of Guangdong Women and Children Hospital for technical assistance.

\section{Authors' contributions}

Nong YQ designed the study and contributed to article revision. Li SF performed the research and contributed to writing the article. They share first authorship. Liu WJ, Fan L, Chen Y and Huang QW contributed to experiment design and figures performed. Zhang XQ and Zhang QY were responsible for analysis of data. Liu FH designed the work, provided technical guidance and final approved of manuscript. All authors read and approved the final manuscript.

\section{Funding}

This study was supported by Natural Science Foundation of Guangdong Province, China (No. 2016A030313817), Science and Technology Program of Guangzhou, China (201704020217), and the In-Hospital Training Project of the National Natural Science Foundation of Guangdong Women and Children Hospital, China (No. YN2017G10).

\section{Availability of data and materials \\ Not applicable.}

\section{Declaration}

Ethics approval and consent to participate

This study was approved by the Ethics Committee of Guangdong Women and Children Hospital and has been performed in accordance with the principles of Declaration of Helsinki.

\section{Consent for publication}

Not applicable.

\section{Competing interests}

The authors have no conflict of interest to declare.

\section{Author details}

${ }^{1}$ The First Affiliated Hospital of Jinan University, Guangzhou, China.

${ }^{2}$ Department of Reproductive Health and Infertility, Guangdong Women and Children Hospital, Guangzhou, Guangdong, China. ${ }^{3}$ Reproductive Medicine Center, Affiliated Shenzhen City Maternity and Child Healthcare Hospital of Southern Medical University, Shenzhen, China.

Received: 4 September 2020 Accepted: 24 February 2021

Published online: 29 March 2021

References

1. Coughlan C. What to do when good-quality embryos repeatedly fail to implant. Best Pract Res Clin Obstet Gynaecol. 2018;53:48-59.

2. Shohayeb A, El-Khayat W. Does a single endometrial biopsy regimen (S-EBR) improve ICSI outcome in patients with repeated implantation failure? A randomised controlled trial. Eur J Obstet Gynecol Reprod Bio. 2012;164:176-9.

3. Achache $H$, Revel A. Endometrial receptivity markers, the journey to successful embryo implantation. Hum Reprod Update. 2006;12:731-46.

4. Burrows TD, King A, Loke YW. Trophoblast migration during human placental implantation. Hum Reprod Update. 1996;2:307-21. 
5. Cross JC, Werb Z, Fisher SJ. Implantation and the placenta: key pieces of the development puzzle. Science. 1994;266:1508-18.

6. Murray MJ, Lessey BA. Embryo implantation and tumor metastasis: common pathways of invasion and angiogenesis. Semin Reprod Endocrinol. 1999;17: 275-90.

7. Verkman AS, Mitra AK. Structure and function of aquaporin water channels. Am J Physiol Renal Physiol. 2000;278:F13-28.

8. Maggio $\mathrm{M}$, et al. Association of hormonal dysregulation with metabolic syndrome in older women: data from the InCHIANTI study. Am J Physiol Endocrinol Metab. 2007;292:E353-8.

9. Dong C, Aznavoorian S, Liotta LA. Two phases of pseudopod protrusion in tumor cells revealed by a micropipette. Microvasc Res. 1994;47:55-67.

10. Verkman AS. More than just water channels: unexpected cellular roles of aquaporins. J Cell Sci. 2005;118:3225-32.

11. Steffens $\mathrm{S}$, et al. Low dose oral cannabinoid therapy reduces progression of atherosclerosis in mice. Nature. 2005;434:782-6.

12. Xiong $Y$, et al. Expression of aquaporins in human embryos and potential role of AQP3 and AQP7 in preimplantation mouse embryo development. Cell Physiol Biochem. 2013;31:649-58.

13. King LS, Kozono D, Agre P. From structure to disease: the evolving tale of aquaporin biology. Nat Rev Mol Cell Biol. 2004;5:687-98.

14. Ducza E, Csanyi A, Gaspar R. Aquaporins during pregnancy: their function and significance. Int J Mol Sci. 2017;18.

15. Martinez N, Damiano AE. Aquaporins in fetal development. Adv Exp Med Biol. 2017;969:199-212.

16. Escobar J, et al. Expression of aquaporins early in human pregnancy. Early Hum Dev. 2012;88:589-94.

17. Nong $Y Q$, et al. The expression and distribution of aquaporin 3 in mouse embryos before and after vitrification. J Assist Reprod Genet. 2013;30:601-6.

18. Fang $C X$, et al. Heparin-binding epidermal growth factor-like growth factor enhances aquaporin 3 expression and function during mouse embryo implantation. Reprod Sci. 2017;24:463-70.

19. Zhang $\mathrm{Q}$, et al. Expression of $\mathrm{CD} 82$ in human trophoblast and its role in trophoblast invasion. PLoS One. 2012;7:e38487.

20. Alekseev OM, et al. Analysis of gene expression profiles in HeLa cells in response to overexpression or siRNA-mediated depletion of NASP. Reprod Biol Endocrinol. 2009:7:45.

21. Marlar, S., et al., Aquaporin-3 in Cancer. Int J Mol Sci. 2017;18(10).

22. Hara-Chikuma M, Verkman AS. Prevention of skin tumorigenesis and impairment of epidermal cell proliferation by targeted aquaporin-3 gene disruption. Mol Cell Biol. 2008;28:326-32.

23. Hara-Chikuma M, Verkman AS. Aquaporin-3 facilitates epidermal cell migration and proliferation during wound healing. J Mol Med (Berl). 2008; 86:221-31.

24. Huang $Y T$, et al. Identification of estrogen response element in Aquaporin-3 gene that mediates estrogen-induced cell migration and invasion in estrogen receptor-positive breast Cancer. Sci Rep. 2015;5:12484.

25. Chen J, et al. Aquaporin 3 promotes prostate cancer cell motility and invasion via extracellular signal-regulated kinase 1/2-mediated matrix metalloproteinase-3 secretion. Mol Med Rep. 2015;11:2882-8.

26. Hou SY, et al. Aquaporin-3 inhibition reduces the growth of NSCLC cells induced by hypoxia. Cell Physiol Biochem. 2016;38:129-40.

27. Cui D, et al. Aquaporin-3 mediates ovarian steroid hormone-induced motility of endometrial epithelial cells. Hum Reprod. 2018;33:2060-73.

28. Alejandra R, Natalia S, Alicia ED. The blocking of aquaporin-3 (AQP3) impairs extravillous trophoblast cell migration. Biochem Biophys Res Commun. 2018;499(2):227-32.

29. Xiong $\mathrm{G}$, et al. RNA interference influenced the proliferation and invasion of XWLC-05 lung cancer cells through inhibiting aquaporin 3. Biochem Biophys Res Commun. 2017:485(3):627-34.

30. Andrae J, Gallini R, Betsholtz C. Role of platelet-derived growth factors in physiology and medicine. Genes Dev. 2008;22:1276-312.

31. Honda $\mathrm{M}$, et al. Mesothelioma cell proliferation through autocrine activation of PDGF-betabeta receptor. Cell Physiol Biochem. 2012;29:667-74.

32. Schwenke $\mathrm{M}$, et al. Control of human endometrial stromal cell motility by PDGF-BB, HB-EGF and trophoblast-secreted factors. PLoS One. 2013;8:e54336.

33. Jing $Y$, et al. SPARC promotes the proliferation and metastasis of oral squamous cell carcinoma by PI3K/AKT/PDGFB/PDGFRbeta axis. J Cell Physiol. 2019.

\section{Publisher's Note}

Springer Nature remains neutral with regard to jurisdictional claims in published maps and institutional affiliations.

\section{Ready to submit your research? Choose BMC and benefit from:}

- fast, convenient online submission

- thorough peer review by experienced researchers in your field

- rapid publication on acceptance

- support for research data, including large and complex data types

- gold Open Access which fosters wider collaboration and increased citations

- maximum visibility for your research: over $100 \mathrm{M}$ website views per year

At BMC, research is always in progress.

Learn more biomedcentral.com/submissions 